\title{
Laparoscopically assisted colonoscopic polypectomy - viable option for curative surgery in elderly patients
}

\author{
Rokas Račkauskas ${ }^{1}$, Saulius Mikalauskas ${ }^{1}$, Marius Petrulionis ${ }^{1}$, Tomas Poškus ${ }^{1}$, Valdemaras Jotautas ${ }^{1}$, \\ Juozas Stanaitis², Eligijus Poškus ${ }^{1}$, Kęstutis Strupas ${ }^{1}$ \\ ${ }^{1}$ Centre of Abdominal Surgery, Vilnius University, Vilnius, Lithuania \\ ${ }^{2}$ Center of Hepatology and Gastroenterology, Vilnius University, Vilnius, Lithuania
}

Videosurgery Miniinv 2017; 12 (2): 120-124

DOI: https://doi.org/10.5114/wiitm.2017.68138

\begin{abstract}
Introduction: Colorectal cancer $(C R C)$ is the third most common cancer worldwide and the fourth most frequent cause of cancer-related death in the world. CRC screening programs have been widely introduced worldwide, allowing for early detection and removal of precancerous lesions and avoiding major surgical intervention. However, not all polyps are suitable for conventional and advanced colonoscopic polypectomy. Thus, laparoscopically assisted colonoscopic polypectomy (LACP) was introduced to clinical practice as a method of choice for these polyps and adenomas.

Aim: To overlook our experience in laparoscopically assisted colonoscopic polypectomies and evaluate effectiveness and quality of the procedure.

Material and methods: A retrospective analysis of a prospectively maintained database was performed. using the Vilnius University Hospital Santariskiu Klinikos patient database for the period from 2010 to 2016, resulting in 21 cases in which LACP was performed. All procedures were performed using combined laparoscopy and videocolonoscopy techniques. Morphology of adenomas was classified according to the Paris classification during the procedure. Creation of the database was approved by the Lithuanian Bioethics committee.

Results: Twenty-two adenomas were removed from 21 patients, aged $65.33 \pm 8.9$. There was no difference between male and female age, but occurrence of adenomas in females was 2-fold higher. The majority of removed lesions were localized in the cecum and mean size was $27.2 \pm 11.1 \mathrm{~mm}$. The morphology of adenomas was distributed equally between 0-Is, 0-Ip, and 0-IIa, except one, which belonged to 0-III. Histological analysis revealed that tubulovillous adenoma occurrence was 1.4 times higher than tubulous adenoma. There was only one postoperative complication - bleeding from the adenoma resection site, which was managed by conservative means. One patient developed G2 adenocarcinoma at the polyp resection site and was referred for radical surgery.

Conclusions: The LACP is a safe procedure with minimal risk to the elderly patient. Patient follow-up is essential for detection of recurrence.
\end{abstract}

Key words: colonoscopy, laparoscopy, laparoscopically assisted polypectomy, minimally invasive technique.

\section{Introduction}

Colorectal cancer (CRC) is the third most common cancer and the fourth most common cause of cancer death in the world, accounting for $9 \%$ of cancer-related deaths [1-3]. Effective CRC screening programs have been established, mostly based on occult blood test and colonoscopy for detection of early CRC and related potentially precancerous lesions. It has been shown that screening programs with subsequent removal of colorectal polyps and adenomas reduce the CRC rate [2]. However, not all 
benign lesions can be successfully removed using conventional colonoscopy due to their inconvenient location including large bowel flexures, folds and Bauhin's valve, large size of more than $3-4 \mathrm{~cm}$, and also due to the flat shape [4].

Recently, laparoscopically assisted colonoscopic polypectomy (LACP) was introduced for difficult and large benign lesions, thus reducing the need for major surgical intervention [5]. In our hospital, laparoscopically assisted colonoscopic polypectomy has been implemented for 6 years and it has become the standard treatment option for patients with advanced large bowel adenomas.

\section{Aim}

Aim of this study was to overlook our experience in laparoscopically assisted colonoscopic polypectomies and evaluate effectiveness and quality of the procedure.

\section{Material and methods}

A retrospective analysis of a prospectively maintained database was performed using the Vilnius University Hospital Santariskiu Klinikos patient database. We included patients operated on from 2010 to 2016. We identified 21 patients who underwent LACP. The bioethics committee approved the database creation (approval no. L-13-03/1).

All LACPs were performed using an Olympus CF-Q165L (Olympus GmbH, Germany) videocolonoscope. An endoscopic needle was used to inject a solution of saline and methylene blue into the submucosal layer. Different diameters of diathermic loops were used for piecemeal mucosectomies of adenoma. Laparoscopy was performed using an Olympus EXERA 3 (Olympus GmbH, Germany) laparoscope. Pneumoperitoneum was formed using carbon dioxide $\left(\mathrm{CO}_{2}\right)$ gas and abdominal pressure was maintained at $10 \mathrm{~mm} \mathrm{Hg}$. Laparoscopic instruments were inserted and, if present, adhesions were removed and the bowel segment with the lesion was mobilized for better visualization. The part of the colon proximal to the lesion or distal part of the ileum was clamped with soft graspers as described elsewhere [6]. Then colonoscopy was performed using $\mathrm{CO}_{2}$ insufflation. Under active laparoscopic assistance holding, pulling, and sometimes inverting the bowel wall, endoscopic mucosectomy was performed. After the procedure the bowel wall was inspected both visually and using the air leak test. Operating time was measured from the first incision until the closure of the last incision.

All lesions were classified according to the Paris classification and all adenomas were divided into 4 groups: 0-Is, 0-Ip, 0-IIa, and 0-III [7]. All resected adenomas or their pieces were extracted, measured and sent to a pathologist for histological examination.

\section{Statistical analysis}

All data are presented as mean values with standard deviation. Differences between groups were assessed with the $t$-test; values were considered significant when $p<0.05$. All calculations were performed with GraphPad Prism 5 (GraphPad software, Inc, USA) software.

\section{Results}

Twenty-one patients underwent LACP for 22 benign lesions (summary in Table I). Adenoma occurrence was 2 -fold higher in females than in males, accounting for $66.7 \%$ and $33.3 \%$ respectively, $p<0.05$. There was no difference in patient age between females and males, $63.9 \pm 9.8$ vs. $68.1 \pm 6.6$ respectively, $p<0.3$. Mean hospital stay was $3.7 \pm 2.4$ days.

Mean operating time was $84.5 \pm 33.0 \mathrm{~min}$. During all surgical procedures blood loss was absent and there was no bowel perforation after procedures.

Almost half of adenomas were located in the cecum, accounting for 10 (45.4\%), and other adenomas were located in the large intestine almost equally per region. Mean size of resected adenoma was $27.2 \pm 11.1 \mathrm{~mm}$. According to the Paris classification, all benign lesions were distributed equally between the first three groups, 7 in each, except one, which belonged to $0-1$ II.

A dedicated colonic pathologist performed histological analysis on each specimen. Tubulovillous adenoma occurrence was 1.4 times higher than tubulous adenoma. Moreover, we found that 6 of 13 tubulovillous adenomas had a high or low-grade dysplasia vs. 2 of 9 in tubulous adenoma, $46.2 \%$ vs. $22.2 \%$.

There was only one complication (4.8\%) - bleeding from the lower intestinal tract on the second day after the procedure, which was managed conservatively with no surgical intervention.

Only 10 out of 22 (45.5\%) patients were screened for recurrent adenomas at our hospital; $30 \%$ of them had recurrent adenomas at the same location of the 


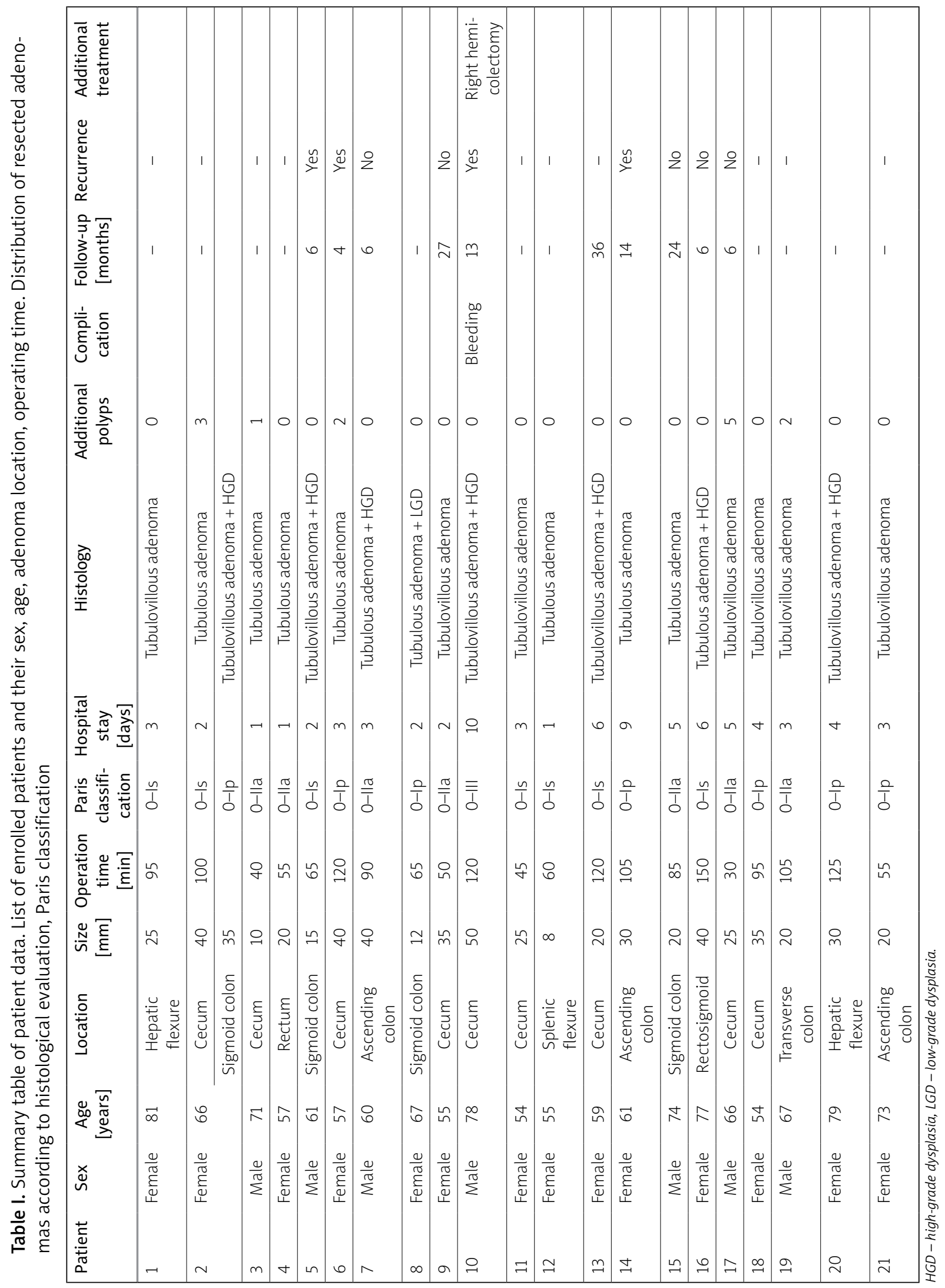


initial polypectomy, which were removed during the same colonoscopy. One (10\%) patient had a recurrent lesion after 12 months of follow-up, which histologically showed G2 adenocarcinoma, and the patient underwent right hemicolectomy subsequently. The final histological stage was T1NOMO.

\section{Discussion}

We found that LACP is a safe and effective method for advanced colorectal adenomas with difficult location. Mean age was similar to other conducted studies, but we found that the gender distribution was different, with a predominance of female patients $[7,8]$.

Hybrid laparoscopic-endoscopic procedures usually are lengthy, compared to conventional colonoscopy, due to combined procedures that must be performed. Our experience did not differ from other studies, resulting in operating time of around $1.5 \mathrm{~h}[8,9]$.

Minimally invasive methods are advantageous compared to open surgery for elderly patients because of the high morbidity rate after procedures and long hospitalization. Arezzo et al. in a systematic review of 11 studies found that the mean complication rate after LACP was $7.9 \%$. In our study we had a complication rate of $4.8 \%$ and mean hospital stay length was comparable with other studies $[8,10]$.

Large colorectal polyps are one of the indications for LACP. The literature showed that resected polyp size can range from $1.0 \mathrm{~cm}$ to $7.0 \mathrm{~cm}[11,12]$. In our experience the mean polyp size was $27.8 \mathrm{~mm}$, with the largest reaching $5.0 \mathrm{~cm}$ in diameter, but polyp location was a more influential factor for LACP. We found that the majority of resected polyps were located in the cecum. These findings correlate with other studies, but some papers report that the majority of resected polyps occur equally in the right and transverse colon [6, 13].

Before removal of all polyps, they were morphologically classified, according to the Paris classification [7]; we found equal distribution in sessile, pedunculated and superficial elevated polyps. These findings differ from previous papers, which reported that almost all polyps that were removed were sessile [13]. Our results clearly show that LACP is safe for heterogeneous polyp removal without the risk of perforating the bowel.

Regarding histology, many papers report that the majority of resected polyps were tubular and tubulovillous adenomas with different frequency $[6,13]$. The percentage of polyps with high-grade dysplasia in resected polyps ranges up to $33.3 \%$ [14]. In our experience, however, we found that tubulovillous adenomas were dominating over other histological types, and we also found that high-grade dysplasia occurrence was up to 2-fold higher than low-grade dysplasia and was present in almost half the resected polyps. Histology showed that all polyps were removed radically; however, radicality might be questionable because some of these specimens were resected by a piecemeal method. These findings suggest that LACP is feasible for removal of polyps that otherwise would require a more extended operation. However, some authors prefer laparoscopic local resection of the bowel lesion [15].

A downside of this retrospective analysis was that there was no established protocol for patient follow-up. Only $45.5 \%$ of patients were admitted for colonoscopy follow-up; moreover, the adenoma recurrence rate was almost 10-fold higher than in previous papers $[10,14]$. One tenth of all follow-up patients had adenocarcinoma recurrence in a resected polyp with high-grade dysplasia that was subjected to radical treatment.

A strict follow-up protocol needs to be established, since there is no way of knowing the actual recurrence rate otherwise.

\section{Conclusions}

Laparoscopically assisted colonoscopic polypectomy is a safe procedure with minimal risk of complications and should be used for patients who have advanced, large adenomas in difficult locations. Patient follow-up is essential for detection of recurrence.

\section{Conflict of interest}

Saulius Mikalauskas, Rokas Račkauskas, Tomas Poškus, Eligijus Poškus, Valdemaras Jotautas, and Kęstutis Strupas declare no conflict of interest.

\section{References}

1. World Cancer Research Fund and American Institute for Cancer Research Food, Nutrition, Physical Activity, and the Prevention of Cancer: A Global Perspective. Washington, DC: American Institute for Cancer Research; 2007.

2. Boyle P, Langman JS. ABC of colorectal cancer: epidemiology. BMJ 2000; 321: 805-8. 
3. Torre LA, Bray F, Siegel RL, et al. Global cancer statistics, 2012. CA Cancer J Clin 2015; 65: 87-108.

4. Ommer A, Limmer J, Möllenberg H, et al. Laparoscopic-assisted colonoscopic polypectomy: indications and results. Zentralbl Chir 2003; 128: 195-8.

5. Prohm P, Weber J, Bönner C. Laparoscopic-assisted coloscopic polypectomy. Dis Colon Rectum 2001; 44: 746-8.

6. Franklin ME Jr, Diaz EJA, Abrego G, et al. Laparoscopic assisted colonoscopic polypectomy: the Texas Endosurgery Institute ex perience. Dis Colon Rectum 2000; 43: 1246-9.

7. Endoscopic Classification Review Group. Update on the Paris classification of superficial neoplastic lesions in the digestive tract. Endoscopy 2005; 37: 570-8.

8. Voloyiannis T, Snyder MJ, Bailey RR, et al. Management of difficult colon polyps referred for resection: resect or rescope? DCR 2008; 51: 292-5.

9. Goh C, Burke JP, NcNamara DA, et al. Endolaparoscopic removal of colonic polyps. Colorectal Dis 2004; 16: 271-5.

10. Arezzo A, Passera R, Migliore M, et al. Efficacy and safety of laparo-endoscopic resections of colorectal neoplasia: a systematic review. United Eur Gastroenterol I 2015; 3: 514-22.

11. Lee SW, Garrett KA, Shin JH, et al. Long-term outcomes of patients undergoing combined endolaparoscopic surgery for benign colon polyps. Dis Colon Rectum 2013; 56: 869-73.

12. Yan J, Trencheva K, Lee SW, et al. Treatment for right colon polyps not removable using standard colonoscopy: combined laparoscopic-colonoscopic approach. Dis Colon Rectum 2011; 54: 753-8.

13. Lascarides C, Buscaglia JM, Denoya PI, et al. Laparoscopic right colectomy versus laparoscopic-assisted colonoscopic polypectomy for endoscopically unresectable polyps: a randomised controlled trial. Colorectal Dis 2016. Epub 2016/04/03.

14. Crawford AB, Yang I, Wu RC, et al. Dynamic article: combined endoscopic-laparoscopic surgery for complex colonic polyps: postoperative outcomes and video demonstration of 3 key operative techniques. Dis Colon Rectum 2015; 58: 363-9.

15. Dulskas A, Samalavicius NE, Gupta RK, Zabulis V. Laparoscopic colorectal surgery for colorectal polyps: single institution experience. Videosurgery Miniinv 2015; 10: 73-8.

Received: 15.03.2017, accepted: 14.04.2017. 